\title{
Procalcitonin for reduced antibiotic
}

\section{exposure in ventilator-associated pneumonia: a randomised study}

\author{
D. Stolz*\#, , N. Smyrnios", P. Eggimann+, H. Pargger ${ }^{\S}$, N. Thakkar", M. Siegemund ${ }^{\S}$, \\ S. Marsch ${ }^{\dagger}$, A. Azzola**, J. Rakic*, B. Mueller ${ }^{\# \#}$ and M. Tamm*
}

ABSTRACT: In patients with ventilator-associated pneumonia (VAP), guidelines recommend antibiotic therapy adjustment according to microbiology results after $72 \mathrm{~h}$. Circulating procalcitonin levels may provide evidence that facilitates the reduction of antibiotic therapy.

In a multicentre, randomised, controlled trial, 101 patients with VAP were assigned to an antibiotic discontinuation strategy according to guidelines (control group) or to serum procalcitonin concentrations (procalcitonin group) with an antibiotic regimen selected by the treating physician. The primary end-point was antibiotic-free days alive assessed 28 days after VAP onset and analysed on an intent-to-treat basis.

Procalcitonin determination significantly increased the number of antibiotic free-days alive 28 days after VAP onset (13 (2-21) days versus 9.5 (1.5-17) days). This translated into a reduction in the overall duration of antibiotic therapy of $27 \%$ in the procalcitonin group $(p=0.038)$. After adjustment for age, microbiology and centre effect, the rate of antibiotic discontinuation on day 28 remained higher in the procalcitonin group compared with patients treated according to guidelines (hazard rate $1.6,95 \% \mathrm{Cl}$ 1.02-2.71). The number of mechanical ventilation-free days alive, intensive care unit-free days alive, length of hospital stay and mortality rate on day 28 for the two groups were similar.

Serum procalcitonin reduces antibiotic therapy exposure in patients with ventilator associated pneumonia.

KEYWORDS: Antibiotic therapy, biomarker, procalcitonin, ventilator-associated pneumonia

$\mathbf{V}$ entilator associated pneumonia (VAP) is the leading cause of death from nosocomial infection in the USA [1-3]. The mortality attributable to VAP has been reported to range between $24 \%$ and $50 \%$ [4, 5]. The estimated excess cost for an episode of VAP can be as high as $\$ 40,000$ per patient $[5,6]$. Based on an estimate of 250,000 cases of VAP annually, the cost of treatment easily approaches $\$ 10$ billion in the USA alone [7].

At the present time, there is no gold standard for the diagnosis of VAP and the optimal approach for diagnosing VAP remains to be defined [8-10]. Prompt initiation of broad-spectrum antibiotic therapy is a cornerstone of treatment of VAP because even relatively short delays in administering adequate antibiotic therapy are associated with an increased mortality rate [11-13]. Unfortunately, exposure to antibiotics, especially for $\geqslant 7$ days, has been associated with subsequent emergence of infection with antibiotic-resistant bacteria and worse outcome [14-18]. Clinicians managing patients with suspected VAP should employ antimicrobial treatment strategies that minimise prolonged and potentially unnecessary antibiotic exposure to curtail resistance [19]. In this context, the concept of de-escalation therapy is emerging as an effective strategy to reduce the development of bacterial resistance in patients treated for VAP $[10,20]$. Appropriately shortening the treatment duration is an important aspect of decreasing antibiotic-associated costs, minimising selection pressures for resistant organism in the ICUs and improving outcomes [8]. The most recent treatment guidelines by the American Thoracic Society (ATS) and Infectious Disease Society of America (IDSA) have put forth a strategy to address early stoppage of antibiotics [21]. Accordingly, upon suspicion of VAP, lower respiratory tract sampling for cultures are obtained and empiric broad-spectrum antibiotics started. A systematic re-evaluation at 48 to $72 \mathrm{~h}$, should allow for strongly considering stopping

\section{AFFILIATIONS}

${ }^{*}$ Clinic of Pulmonary Medicine and Respiratory Cell Research, ${ }^{\S}$ Division of Anaesthesiology and Surgical Intensive Care Medicine, ${ }^{f}$ Division of Medical Intensive Care Medicine, University Hospital, Basel, ${ }^{+}$Dept of Adult Critical Care Medicine, University Hospital, Lausanne,

${ }^{* *}$ Dept of Pulmonary Medicine, Regional Hospital Civico, Lugano,

\#\#Dept of Internal Medicine, Kantonsspital, Aarau, Switzerland,

\#The Harvard School of Public Health, Boston, and

"Division of Pulmonary, Allergy and Critical Care Medicine, UMass Memorial Medical Center, Worcester, MA, USA.

CORRESPONDENCE

D. Stolz

University Hospital

Petersgraben 4

CH-4031 Basel

Switzerland

E-mail:dstolz@uhbs.ch

Received:

March 312009

Accepted after revision:

Sept 022009

First published online:

Sept 242009

European Respiratory Journal

Print ISSN 0903-1936

Online ISSN 1399-3003 
antibiotics if the patient is improving and cultures are negative. Should the culture results be positive and the patient has improved, then antibiotics being administered are tailored to the culture results, including potential for monotherapy [10]. Regrettably, this treatment algorithm often is not adhered to in daily clinical practice [19]. Some authors have suggested that inability to obtain uncontaminated lower respiratory tract secretions and the pressures to treat an increasingly ill population are some of the factors that limit adherence to this approach [22].

Procalcitonin, the precursor molecule of calcitonin, is upregulated in severe bacterial infections and sepsis [23, 24]. Procalcitonin may be considered as a valuable biomarker for the diagnosis of bacterial infections, including VAP, where its levels are correlated with outcome $[25,26]$. Its usefulness for guiding antibiotic therapy has been shown in patients admitted for community-acquired respiratory tract infections [27-29]. It has been recently suggested that serial measurements of procalcitonin allow a reduction in the duration of antibiotic therapy without resulting in more adverse outcomes in critically ill patients with severe sepsis and septic shock [30]. Despite the growing body of literature in favour of this biomarker [27-31], it is unknown whether procalcitonin enables a refinement in the current ATS/IDSA antibiotic stoppage strategy in VAP. Therefore, we undertook this multicentre, randomised trial to determine if procalcitonin serum evaluation reduces antibiotic exposure in patients with clinically diagnosed VAP with a similar clinical and laboratory outcome.

\section{METHODS}

\section{Setting and study population}

This is a multinational, randomised, controlled open intervention trial in patients with VAP requiring treatment at an intensive care unit (ICU) performed in seven ICUs (UMass Memorial Medical Center, Worcester, MA, USA; University Hospital Lausanne, Switzerland and University Hospital, Basel, Switzerland). The results are reported following the consolidated standards of reporting trials statement (fig. 1) [32]. We compared antibiotic therapy duration in patients treated according to current guidelines (control group) with patients in who antibiotic treatment was guided by serum procalcitonin levels (procalcitonin group). The study was approved by the institutional review boards of all participating institutions and registered in the Current Controlled Trials Database as "ProVAP"-Study (ISRCTN61015974) [33]. Written informed consent was obtained from all included patients or their legal representatives. All data were held and analysed by the authors.

ICU patients intubated for mechanical ventilation for $\geqslant 48 \mathrm{~h}$ were eligible for the study if they met all the following criteria: 1) $>18$ yrs; 2) clinically diagnosed VAP as defined by the ATS guidelines (new or persistent infiltrate on chest radiography associated with at least two of the following: purulent tracheal secretions, temperature $>38^{\circ} \mathrm{C}$ or, leukocyte count $>11,000 \mu \mathrm{L}$ or $<3,000 \mu \mathrm{L}$ ) [21]. Patients were excluded it they 1) were pregnant; 2) were enrolled in another trial; 3) had received immunosuppressants or long-term corticosteroid therapy ( $\geqslant 0.5 \mathrm{mg} \cdot \mathrm{kg}^{-1} \cdot \mathrm{day}^{-1}$ for $\left.>1 \mathrm{month}\right) ; 4$ ) were severely immunosuppressed, including acquired immunodeficiency syndrome; and 5) had a coexisting extrapulmonary infection diagnosed between day 1 and 3 requiring antibiotic therapy for $>3$ days.

\section{Randomisation}

Patients were randomly assigned to one of the two approaches after agreement of the attending physician. Randomisation was through arbitrary allocation to one of the two treatment assignments based on sealed, opaque envelopes. Block size was 20 envelopes. Treating physicians were not aware of envelope contents before randomisation. In both groups, antibiotic therapy was started at inclusion (day 0), according to the decision of the attending physician, who was unaware of procalcitonin levels. Educational sessions, posters and instruction cards highlighting the current standard antibiotic de-escalation strategy according to the ATS were provided to attending physicians and those responsible for patient's treatment decisions [21]. Thereby, particular emphasis was set on the recommendations regarding the stoppage of antibiotics according to microbiology results after $72 \mathrm{~h}$ and duration of antibiotic therapy as suggested by the ATS [21].

After $72 \mathrm{~h}$ (day 2), daily procalcitonin levels were notified to the attending physician, automatically and/or by personal communication, for patients randomised to the procalcitonin group. Thereafter, the physician in charge was advised to classify the patients into four groups, according to the probability of ongoing bacterial infection [27]. A procalcitonin level of $<0.25 \mu \mathrm{g} \cdot \mathrm{L}^{-1}$ suggested the absence of VAP and discontinuation of antibiotics was strongly encouraged. A procalcitonin level between 0.25 and $0.5 \mu \mathrm{g} \cdot \mathrm{L}^{-1}$ or a decrease by $\geqslant 80 \%$ compared with day 0 indicated that bacterial infection was unlikely and reduction or discontinuation of antibiotics was encouraged. A procalcitonin level $\geqslant 0.5 \mu \mathrm{g} \cdot \mathrm{L}^{-1}$ or decrease by $<80 \%$ compared with day 0 was considered to indicate unresolved bacterial infection and reduction or discontinuation of antibiotics was discouraged. A procalcitonin level of $>1 \mu \mathrm{g} \cdot \mathrm{L}^{-1}$ strongly suggested unresolved bacterial infection and antibiotic discontinuation was strongly discouraged. After day 2, evaluation of procalcitonin levels was

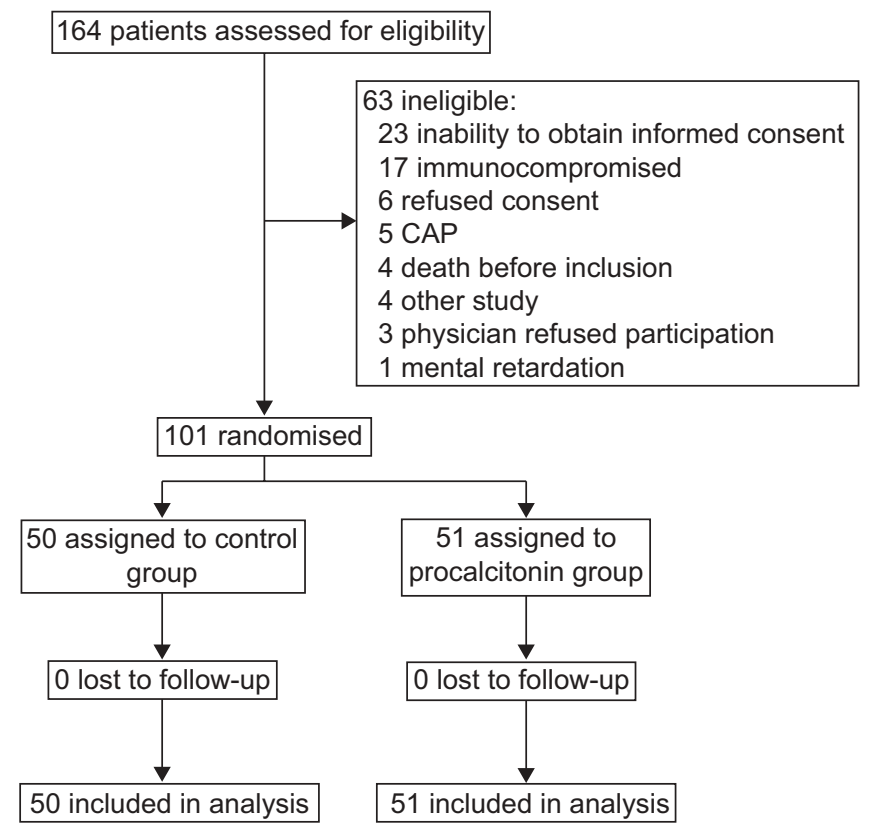

FIGURE 1. Trial profile. CAP: community-acquired pneumonia 
performed by comparing daily procalcitonin levels with the immediately previous value. Re-evaluation of the clinical status and measurement of serum procalcitonin levels were performed daily for both groups up to day 10 after inclusion. Attending physicians responsible for patients in the control group remained unaware of procalcitonin throughout the study.

\section{Measurement of serum procalcitonin}

Measurements were done using a time-resolved amplified cryptate emission technology assay (Kryptor ${ }^{\circledR}$ PCT, Brahms AG, Hennigsdorf, Germany) [27] with a functional assay sensitivity of $0.06 \mu \mathrm{g} \cdot \mathrm{L}^{-1}$, approximately four-fold above mean normal levels [34]. Assay time is $<20 \mathrm{~min}$ and results were routinely available within $1 \mathrm{~h}$.

\section{Antibiotic treatment}

Drug selection was left to the discretion of the treating physicians, including any adaptation considered necessary as a function of clinical, laboratorial, radiological or microbiological results, including bacteriology susceptibility patterns. Upon inclusion in the study and before initiation of antibiotic therapy, sampling of respiratory secretions (endotracheal aspirates, bronchoalveolar lavage, or protected brush specimens) was strongly recommended. The attending and resident physicians in participating ICUs were periodically re-educated on standard antibiotic reduction strategy recommendations [21]. In all situations, treating physicians retained control of antibiotic treatment and were empowered to refrain from antibiotic discontinuation if the duration of antibiotic treatment was considered inadequate or a patient's condition deteriorated.

\section{Baseline assessment and follow-up}

At the time of enrollment the following information was recorded from each subject: age, sex, pre-existing comorbidities, severity of the underlying medical condition(s), primary reason for initiating mechanical ventilation, duration of prior mechanical ventilation, use of any antibiotics within 14 days of VAP onset, presence of infiltrates on chest radiography, fever $\geqslant 38^{\circ} \mathrm{C}$, leukocytosis or leukopenia, purulent tracheal secretions, body temperature, heart rate, mean arterial pressure, oxygen saturation, ratio of arterial oxygen tension $\left(\mathrm{Pa}_{\mathrm{a}} \mathrm{O}_{2}\right)$ to inspiratory oxygen fraction $\left(\mathrm{FI}, \mathrm{O}_{2}\right)$, leukocyte counts and serum procalcitonin values (table 1$)$.

The following indices were calculated: clinical pulmonary infection score (CPIS) score, simplified acute physiologic score (SAPS) II; organ dysfunction and/or infection score (ODIN) and; sepsis-related organ failure assessment score (SOFA).

During the 28 day follow-up period the following information was recorded: body temperature; heart rate; mean arterial pressure; $\mathrm{Pa}_{\mathrm{a}} \mathrm{O}_{2} ; \mathrm{Pa}_{\mathrm{a}} \mathrm{O}_{2} / \mathrm{Fi}, \mathrm{O}_{2}$; CPIS; SOFA; leukocyte counts and serum procalcitonin values for 10 consecutive days; mechanical ventilation status and antibiotic use daily throughout the 28-day study period; vital signs; leukocyte counts and SOFA scores at days 14, 21 and 28; and the patient's status at discharge from the hospital. Any antibiotic use was recorded daily up to day 28. The number of antibiotic-agent days, i.e. number of antimicrobial agents per day $\times$ number of days) was also documented. We calculated the number of antibioticfree days alive as the number of days during the 28 days after living patients had been randomised and had not received any antibiotics, as previously described [16]. VAP-related clinical and/or radiological deterioration was defined as an increase in CPIS of more than two points.

\section{Outcome measures}

The primary end-point was the number of antibiotic-free days alive assessed 28 days after enrollment in the study. Any antibiotic exposure after inclusion, i.e. total antibiotic exposure days and total antibiotic-agent days, regardless of indication, was taken into account for antibiotic exposure analyses. Antibiotic therapy discontinuation related to death was considered a censored event.

Secondary end-points were the number of mechanical ventilation-free days, the number of ICU-free days alive, the evolution of the signs and symptoms potentially linked to pulmonary infection, $\mathrm{Sa}, \mathrm{O}_{2}, \mathrm{~Pa}_{2} \mathrm{O}_{2} / \mathrm{Fi}_{\mathrm{i}} \mathrm{O}_{2}$, the evolution of the SOFA, ODIN and CPIS scores, length of hospital stay, the VAP-related clinical deterioration rate and overall mortality at 28 days. Patients discharged prior to the end of the study (28 days) were followed-up at home or in the post-acute institution for the outcomes of interest.

\section{Statistical analysis}

The trial was designed to demonstrate the superiority of the algorithm incorporating procalcitonin in terms of increasing the number of antibiotic-free days in patients alive within 28 days of inclusion in the study. The sample size was calculated based on the antibiotic-use days in a prior study [16]. Considering 13 antibiotic-free days in the control group and 18 antibiotic-free days in the procalcitonin group, a sample size of 84 patients (42 per group) was necessary to detect a significant difference in antibiotic-free days alive between both groups with a power of $90 \%$ and an $\alpha$ error of 0.05 using a two-tailed test. Assuming $8 \%$ lost to follow-up, we planned the inclusion of 100 subjects.

Discrete variables are expressed as counts (\%) and continuous variables as mean $\pm \mathrm{SD}$ or median (interquartile range (IQR)). End-points were predefined and analysed on the basis of intention-to-treat. Comparability of the control group and the procalcitonin group was analysed by Chi-squared test or Fisher's exact test for categorical variables and nonparametric MannWhitney U-test or unpaired t-test for continuous variables, as appropriate. Cumulative-events curves were estimated with the Kaplan-Meier method. Time to discontinuation of antibiotic treatment was compared between the two study groups by use of the log-rank test. Cox proportional hazards regression analysis was used to evaluate the occurrence of events on day 28, adjusting by age, microbiology of respiratory samples and centre effect ("a priori decision").

\section{RESULTS}

\section{Baseline characteristics of the patients}

During the study period, of the 164 patients with VAP screened for eligibility, 101 were eligible and randomised into the procalcitonin group $(n=51)$ or into the control group $(n=50)$ (fig. 1). The clinical characteristics of these 101 patients at baseline were similar, except that the ODIN score was slightly higher $(p=0.042)$ for the control group (table 1$)$. Three fourths of the patients had received antibiotics within 14 days prior to inclusion in the study, without a significant difference between the groups $(p=0.119)$. At inclusion, serum procalcitonin levels 
TABLE 1 Baseline characteristics of the 101 patients randomised to the control or procalcitonin group

Characteristic

Subjects $n$

Age yrs (min-max)

Male

Admission category

Medical

Emergency surgery

Elective surgery

Origin

Home

Other institution

\section{Coexisting illnesses}

Coronary artery disease

Hypertensive heart disease

Congestive heart failure

Renal dysfunction

Liver disease

Diabetes mellitus

Chronic obstructive pulmonary disease

Neoplastic disease

Substance abuse

Reason for mechanical ventilation

Cardiovascular failure

Acute respiratory failure

Trauma

Neurologic failure

Sepsis

Miscellaneous

Duration of MV before VAP onset days

Antimicrobial therapy 14 days before VAP

Duration of antimicrobial therapy 14 days before VAP days

Criteria for VAP diagnosis

Infiltrate on chest radiograph

Fever $\geqslant 38^{\circ} \mathrm{C}$

Leukocytosis or leukopenia

Purulent tracheal secretions

CPIS $\geqslant 6$

$\mathrm{Fi}, \mathrm{O}_{2} / \mathrm{Pa}, \mathrm{O}_{2}<200$

Shock

Positive blood cultures within $48 \mathrm{~h}$

Diagnostic score ${ }^{\#}$

SAPS ॥

ODIN

SOFA

Clinical and laboratorial findings

Body temperature ${ }^{\circ} \mathrm{C}$

Heart rate bpm

Mean arterial pressure $\mathrm{mmHg}$

$\mathrm{Sa}, \mathrm{O}_{2} \%$

$\mathrm{Pa}_{1} \mathrm{O}_{2} / \mathrm{Fi}_{1} \mathrm{O}_{2} \mathrm{mmHg}$

Leukocyte count $\mu \mathrm{L}$

Procalcitonin $\mu \mathrm{g} \cdot \mathrm{L}^{-1}$

Control group

Procalcitonin group

\section{0}

$59(18-83)$

37 (74)

26 (52)

20 (40)

3 (6)

26 (52)

24 (48)

4 (8)

8 (16)

27 (54)

7 (14)

3 (6)

13 (26)

11 (22)

5 (10)

8 (16)

16 (32)

29 (58)

13 (26)

12 (24)

15 (30)

4 (8)

6 (4-10)

41 (82)

5 (1-9.5)

50 (100)

30 (60)

33 (66)

28 (56)

47 (94)

24 (48)

12 (24)

18 (36)

$45 \pm 14$

$2.3 \pm 1.0$

$8.2 \pm 3.4$

$38.2 \pm 1.2$

$91 \pm 23$

$79 \pm 16$

$97 \pm 3$

$203 \pm 97$

$13.3 \pm 5.9$

$0.73(0.21-2.36)$
51

53 (21-88)

38 (75)

27 (53)

23 (45)

1 (2)

35 (69)

16 (31)

9 (18)

8 (16)

21 (41)

9 (18)

4 (8)

10 (20)

8 (16)

3 (6)

5 (10)

$16(31)$

24 (47)

20 (39)

18 (35)

10 (20)

6 (12)

6 (3-7)

$35(69)$

$3(0-8)$

51 (100)

26 (51)

32 (63)

31 (61)

43 (84)

$25(50)$

11 (22)

14 (28)

$42 \pm 13$

$1.9 \pm 0.9$

$7.3 \pm 3.4$

$37.9 \pm 0.9$

$88 \pm 18$

$81 \pm 16$

$96 \pm 4$

$218 \pm 114$

$12.0 \pm 6.6$

$0.66(0.22-2.69)$

Data are presented as $n(\%)$, median (interquartile range) or mean \pm SD, unless otherwise stated. MV: medical ventilation; VAP: ventilator-associated pneumonia; CPIS: clinical pulmonary infection score; $\mathrm{Fi}, \mathrm{O}_{2}$ : inspiratory oxygen fraction; $\mathrm{Pa}_{1} \mathrm{O}_{2}$ : arterial oxygen tension; SAPS II: simplified acute physiologic score $\mathrm{I}$; ODIN: organ dysfunction and/or infection; SOFA: sepsis-related organ failure assessment; $\mathrm{Sa}, \mathrm{O}_{2}$ : arterial oxygen saturation. There were no other significant differences between the groups with respect to any other characteristic. Because of rounding; percentages may not sum to 100 . The conversion factor for procalcitonin is as follows: $\mu \mathrm{g} \cdot \mathrm{L}^{-1} \times 0.161: \mathrm{nmol} \cdot \mathrm{L}^{-1}$. ${ }^{\#}: \mathrm{ODIN} \mathrm{p}=0.042$. 
were $<0.25 \mu \mathrm{g} \cdot \mathrm{L}^{-1}$ in 29 patients (15 in the control group and 14 in the procalcitonin group; $\mathrm{p}=0.82), 0.25$ to $0.499 \mu \mathrm{g} \cdot \mathrm{L}^{-1}$ in 13 patients (four in the control group and nine in the procalcitonin group; $\mathrm{p}=0.23$ ) and $\geqslant 0.5 \mu \mathrm{g} \cdot \mathrm{L}^{-1}$ in 56 patients ( 30 in the control group and 26 in the procalcitonin group; $p=0.42$ ). Median (IQR) procalcitonin levels in patients pre-treated with antibiotics were $0.73(0.22-2.36) \mu \mathrm{g} \cdot \mathrm{L}^{-1}$, which was not significantly different from patients without antibiotic exposure $0.72 \quad(0.15-$ 2.36) $\mu \mathrm{g} \cdot \mathrm{L}^{-1}$.

\section{Microbiology}

Microbiological investigation of respiratory tract secretion samples was performed in $97(96 \%)$ patients. In 74 (73\%) patients a causative microorganism was identified. The rate of positive bacterial cultures was similar in the control group and the procalcitonin group (76\% versus $71 \%$ ). Microorganisms considered responsible for VAP are listed in table 2 . In both groups the most frequently isolated Gram-negative microorganism was Pseudomonas aeruginosa followed by Klebsiella spp. Most commonly isolated Gram-positive bacteria were methicillin-sensitive Staphylococcus aureus and methicillin-resistant $S$. aureus. Microbiological cultures from others sites were positive in 20 patients (10 control group versus nine procalcitonin group; $\mathrm{p}=0.625)$. In the control group, infection sites were catheter tips (two Staphylococcus non-aureus and one coagulase negative Staphylococcus), spinal fluid (one Streptococcus viridans, one S. aureus), urine (one Enterococcus), wound swabs (one coagulase negative Staphylococcus, one methicilin-resistant S. aureus, one Enterococcus) and blood cultures (one S. aureus). Correspondingly, in the procalcitonin group, infections sites were catheter tips (one Candida albicans), spinal fluid (two Streptococcus pneumonia), urine (one Enterococcus), wound swabs (one Enterococcus, vancomycinresistant Enterococcus, one Enterobacter cloacae and Proteus mirabilis), faeces (one Clostridium difficile), and blood cultures (one Candida albicans).

\section{Primary end-point: reduction of antibiotic exposure}

The median (IQR) number of antibiotic-free days alive within 28 days of diagnosis of VAP was significantly higher in the procalcitonin group than in the control group (13 (2-21) versus 9.5 (1.5-17) days; fig. 2a). This translated into a reduction in the overall duration of antibiotic therapy of $27 \%$ in the procalcitonin group (15 (10-23) versus $10(6-16)$ days; $\mathrm{p}=0.038)$. The total number of antibiotic-agent days was higher in the control group than in the procalcitonin group (1,341 versus 1,077 days). At the end of serial procalcitonin measurements (day 10), the rate of antibiotic discontinuation was significantly higher in the procalcitonin group compared with patients treated according to guidelines (hazard rate 2.235; 95\% CI 1.077-4.64; $\mathrm{p}=0.031$ ). Cox regression-based adjustment of the baseline variables did not substantially modify these findings. The adjusted rate for antibiotic discontinuation of patients in the procalcitonin group versus those in the control group on day 28 was $1.66(95 \% \mathrm{CI}$, 1.02-2.71; fig. 2b) after adjustment for age, respiratory tract culture results and centre effect.

Overall, the median (IQR) duration of antibiotic therapy for VAP was 11 days [6-17]. Antibiotic therapy was continued beyond 7 days in $82 \%$ of subjects in the control group and in $65 \%$ in the procalcitonin group, $(p=0.044 ;$ fig. $3 a)$. In the 28 days following the diagnosis of VAP, antimicrobial monotherapy was

\begin{tabular}{|c|c|c|c|}
\hline \multirow{2}{*}{$\begin{array}{l}\text { TABLE } 2 \\
\text { Organism }\end{array}$} & \multicolumn{3}{|c|}{$\begin{array}{l}\text { Microbiological cultures results from } \\
\text { bronchoscopic and/or endotracheal aspirates } \\
\text { specimens in patients with ventilator-associated } \\
\text { pneumonia according to the treatment algorithm }\end{array}$} \\
\hline & & Control group \# & $\begin{array}{l}\text { Procalcitonin } \\
\text { group }\end{array}$ \\
\hline \multicolumn{4}{|l|}{ Bacilli } \\
\hline \multicolumn{2}{|c|}{ Pseudomonas spp. } & $15(30)$ & $10(20)$ \\
\hline \multicolumn{2}{|c|}{ Acinetobacter spp. } & $0(0)$ & $4(8)$ \\
\hline \multicolumn{2}{|c|}{ Stenotrophomonas maltophilia } & $2(4)$ & $5(10)$ \\
\hline \multicolumn{2}{|c|}{ Escherichia coli } & $8(16)$ & $3(6)$ \\
\hline \multicolumn{2}{|c|}{ Enterobacter } & $6(12)$ & $2(4)$ \\
\hline \multicolumn{2}{|l|}{ Proteus } & $0(0)$ & $1(2)$ \\
\hline \multicolumn{2}{|l|}{ Serratia } & $4(8)$ & $2(4)$ \\
\hline \multicolumn{2}{|l|}{ Klebsiella } & $5(10)$ & $8(16)$ \\
\hline \multicolumn{2}{|l|}{ Citrobacter } & $1(2)$ & $2(4)$ \\
\hline \multicolumn{2}{|c|}{ Morganella morganii } & $0(0)$ & $1(2)$ \\
\hline \multicolumn{2}{|l|}{ Hemophilus } & $5(10)$ & $4(8)$ \\
\hline \multicolumn{2}{|l|}{ Others } & $2(4)$ & $5(10)$ \\
\hline \multicolumn{4}{|l|}{ Cocci } \\
\hline \multicolumn{2}{|l|}{ MSSA } & $10(20)$ & $10(20)$ \\
\hline \multicolumn{2}{|l|}{ MRSA } & $5(10)$ & $5(10)$ \\
\hline \multicolumn{2}{|c|}{ Coagulase-negative Staphylococci } & $1(2)$ & $3(6)$ \\
\hline \multicolumn{2}{|c|}{ Streptococcus } & $1(2)$ & $6(12)$ \\
\hline \multicolumn{2}{|l|}{ Neisseria } & $4(8)$ & $1(2)$ \\
\hline \multicolumn{2}{|c|}{ Enterococcus } & $2(4)$ & $0(0)$ \\
\hline \multicolumn{2}{|l|}{ Others } & $0(0)$ & $2(4)$ \\
\hline
\end{tabular}

Data are presented as $n$ (\%). MSSA: methicillin-sensitive Staphylococcus aureus; MRSA: methicillin-resistant S. aureus. Organisms shown are those isolated at significant concentrations from semi-quantitative endotracheal aspirates or quantitative cultures of protected specimen brush $\left(\geqslant 10^{3} \mathrm{cfu} \cdot \mathrm{mL}^{-1}\right)$ and/or bronchoalveolar lavage fluid $\left(\geqslant 10^{4} \mathrm{cfu} \cdot \mathrm{mL}^{-1}\right)$. Percentages do not sum to 100 because of concomitant isolated microorganisms. ${ }^{*}: n=50 ;{ }^{\bullet}: n=51$.

administered in $22 \%$ of cases (eight control and 14 procalcitonin group); combination therapy with two antimicrobials in $24 \%$ (13 control and 11 procalcitonin group); three antimicrobials in $21 \%$ (12 control and nine procalcitonin group); four antimicrobials in $17 \%$ (eight control and nine procalcitonin group); and five or more antimicrobials in 16\% (nine control and seven procalcitonin group). The most commonly administered antibiotics in both groups included piperacillin/tazobactam in 78 (77\%) patients, vancomycin in $44(44 \%)$, meropenem/imipenem in $31(31 \%)$, levofloxacin in $29(29 \%)$, and amoxicillin/clavulanic acid in 25 (25\%). Appropriate initial empiric antibiotic therapy, defined as a regimen combining an aminoglycoside or a fluoroquinolone plus a betalactam or an antipseudomonal carbepenem, was given in $86 \%$ of the cases without differences between the groups $(p=0.345)$. After $72 h$, combination therapy with two or more drugs could be reduced to monotherapy in $54 \%$ of patients in the procalcitonin group compared with $28.6 \%$ of patients in the control group ( $p=0.008$; fig. $3 b)$.

A microbiologically confirmed VAP influenced maintenance of antibiotic therapy in the control group (hazard rate 2.30, 95\% CI 1.11-4.77) but not in the procalcitonin group (hazard rate 

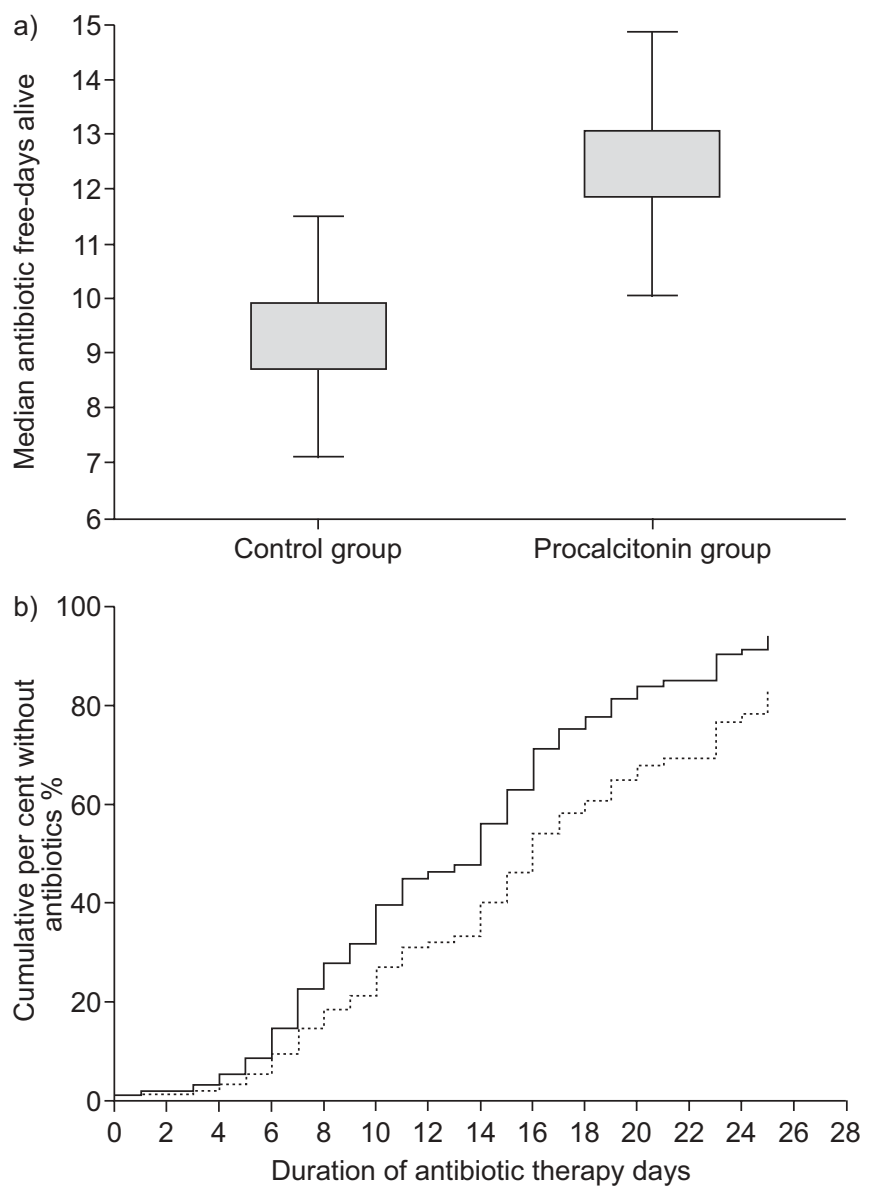

FIGURE 2. a) Median number of antibiotic-free days alive in patients in the control and the procalcitonin group at 28 days after onset of ventilator-associated pneumonia. $p=0.049$. b) Cumulative frequency distribution curve for the time to discontinuation of antibiotics in patients in the control $(\cdots \cdot)$ and the procalcitonin $(-)$ group on day 28. Adjusted by age, respiratory tract culture results and centre effect. $p=0.043$

1.17, 95\% CI 0.59-2.32). Correspondingly, patients with microbiologically diagnosed VAP $(n=73)$ had significantly more median (IQR) antibiotic-free days alive in the procalcitonin group compared with the patients treated in the control group (14 (1-21) versus 7 (0-13.5) days; $\mathrm{p}=0.017)$. Conversely, the number of antibiotic-free days alive were similar in those with negative microbiology $(p=0.563)$. Neither in the procalcitonin group nor in the control group did CPIS scores influence discontinuation of antibiotic therapy ( $p=0.845$ and $p=0.175$ ).

Reasons for antibiotic discontinuation in the control group were clinical and laboratorial improvement $(28 \%, n=14)$, extubation $(16 \%, n=8)$, negative microbiology results $(16 \%$, $\mathrm{n}=8)$ and improvement in CPIS score $(14 \%, \mathrm{n}=7)$. In $10 \%$ $(n=5)$ of the cases antibiotic therapy was maintain beyond 28 days. A total of eight patients deceased while on antibiotic therapy for VAP. Strategies for discontinuation of antibiotic therapy in VAP included cessation of monotherapy $(20 \%$, $n=10)$, dual therapy $(14 \%, n=7)$, or triple therapy $(2 \%, n=1)$, de-escalation from triple to dual therapy $(16 \%, \mathrm{n}=8)$, deescalation from triple or dual to monotherapy $(22 \%, \mathrm{n}=11)$ or adjustment of antibiotics without reduction of the number of
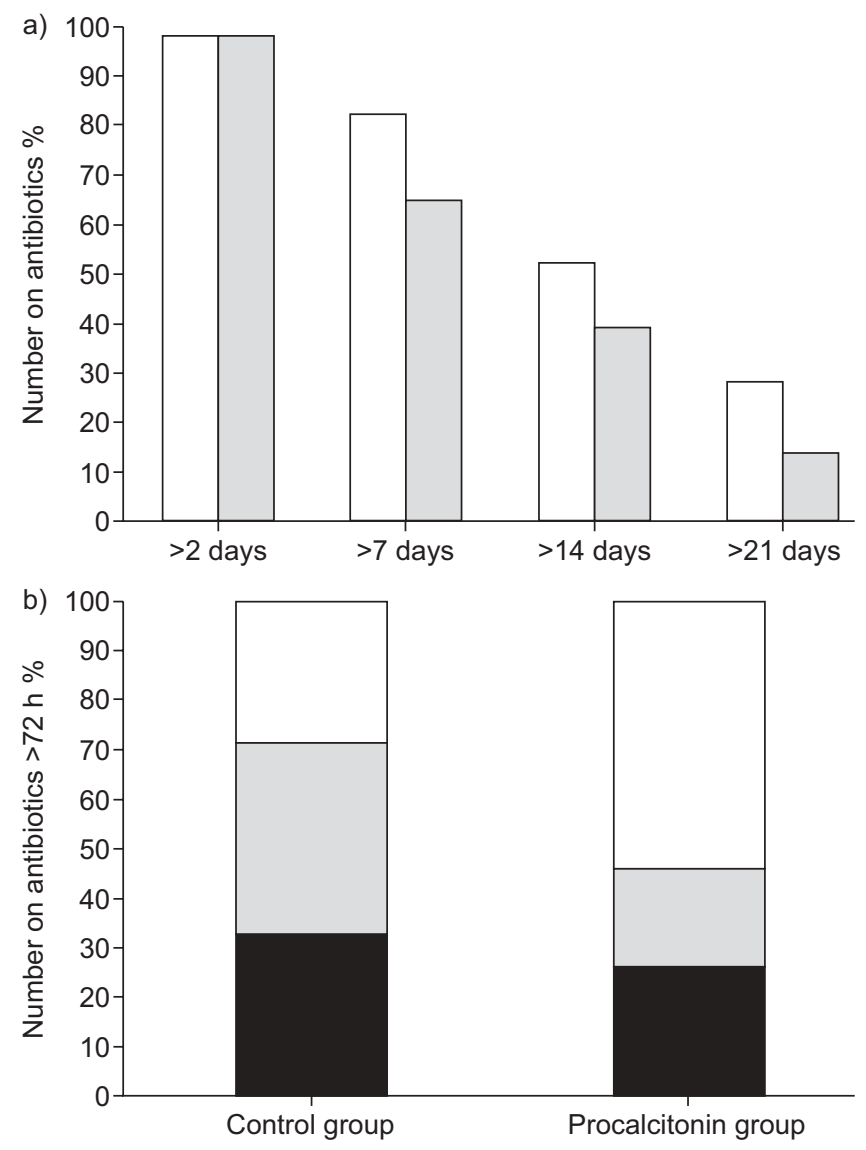

FIGURE 3. a) Duration of antibiotic therapy. Percentage of patients in control $(\square)$ and procalcitonin $(\square)$ group on antibiotics beyond 2, 7, 14 and 21 days. b) Antibiotic reduction profile. Percentage of patients in control group and procalcitonin group on monotherapy and combination therapy with one $(\square)$, two $(\square)$ or three or more $(\square)$ antibiotics after 72 h. $p=0.008$.

antibiotic agents $(26 \%, \mathrm{n}=13)$. Median time to cessation of monotherapy was 9 days (5-12) and to de-escalation from dual to monotherapy was 2 days (1-5). In the procalcitonin group, a total of eight patients with improved CPIS scores received prolonged antibiotic treatment despite low procalcitonin values. In four $(8 \%)$ cases, positive blood cultures with Gram negative bacilli were observed and in another four $(8 \%)$ cases, treating physicians decided to prolong antibiotic treatment due to documented pulmonary infection with Gram negative bacilli (Acinetobacter baumanii, Pseudomonas aeruginosa, Stenotrophomonas maltophilia, and Klebsiella). Duration of antibiotic therapy for VAP was significantly longer in these patients (15 (14-19) versus 7 (4-16) days; $p=0.023)$. The incidence of acquired or persistent microorganisms up to 28 days after VAP onset did not differ between randomised groups ( 16 versus 13 days; $\mathrm{p}=0.5149$; table 3 ).

\section{Secondary end-points: clinical and laboratory outcome}

None of the secondary outcome events (table 4) or physiologic changes seen from day 1 through day 28 differed significantly between patients in the control and procalcitonin groups (fig. 4). The two groups had a similar median (IQR) number of mechanical ventilation and ICU-free days alive. In both groups, 


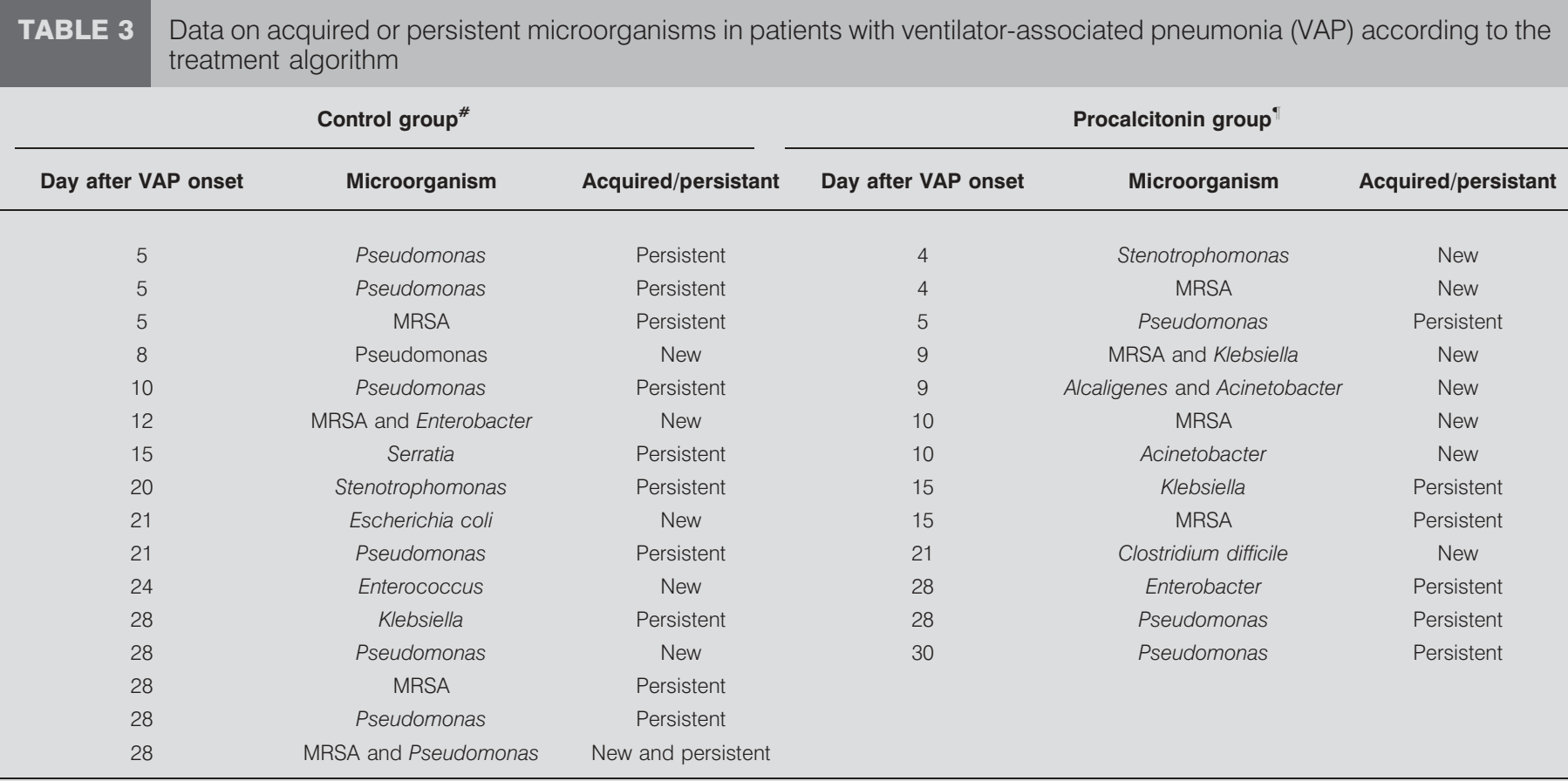

MRSA: methicillin-resistant Staphylococcus aureus. Organisms shown are those isolated at significant concentrations from semi-quantitative endotracheal aspirates or quantitative cultures of protected specimen brush $\left(\geqslant 10^{3} \mathrm{cfu} \cdot \mathrm{mL}^{-1}\right)$ and/or bronchoalveolar lavage fluid $\left(\geqslant 10^{4} \mathrm{cfu} \cdot \mathrm{mL}^{-1}\right)$. ${ }^{*}: \mathrm{n}=50 ;{ }^{\bullet}: \mathrm{n}=51$.

TABLE 4 Secondary study outcomes in patients with ventilator-associated pneumonia (VAP) according to the treatment algorithm

Control group $^{\#} \quad$ Procalcitonin group

p-value

MV-free days alive, days 1-28

All patients

Nonfermenting GNB

MRSA

Other bacteria

No bacteria

ICU-free days alive, days 1-28

All patients

Nonfermenting GNB

MRSA

Other bacteria

No bacteria

\section{Length of hospital stay days}

All patients

Nonfermenting GNB

MRSA

Other bacteria

No bacteria

VAP-related clinical deterioration days $1-28^{+}$

Discharge home days 1-28

Discharge to another institution days $1-28$

Death from all causes days 1-28

In-hospital mortality

$$
\begin{gathered}
19(8.5-22.5) \\
15(7-20) \\
15(0-22) \\
19(1.8-24.8) \\
21.5(18.8-23.3)
\end{gathered}
$$

$8.5(0-18)$

$0(0-11)$

$15(2-17)$

$7.5(1-17.8)$

18 (1.5-20)

26 (16.8-22.3)

34 (26-46)

28 (17-41)

24 (16.5-32.5)

28.5 (16-38)

7 (14)

3 (6)

32 (64)

12 (24)

14 (28)
$21(2-24)$

$12(0.3-23)$

14 (6-21)

$22(15.5-24.5)$

23 (3.5-27)

$10(0-18)$

$4(0-13.5)$

$9(1.5-15)$

14 (7.5-20)

$10(1 .-19.5)$

$26(7-21)$

$31(13-35.5)$

26 (23.5-37.5)

$21.5(14-28)$

29 (9.5-33)

5 (10)

5 (10)

35 (69)

8 (16)

10 (20)
0.455

0.867

1

0.284

0.563

0.526

0.683

0.548

0.139

0.554

0.153

0.130

1.0

0.442

0.343

0.759

0.479

0.509

0.327

0.322

Data are presented as median (interquartile range) and $\mathrm{n}(\%)$, unless otherwise stated. MV: mechanical ventilation; GNB: Gram-negative bacilli; MRSA: methicillinresistant Staphylococcus aureus; ICU: intensive care unit. $\#: n=50 ;{ }^{\bullet}: n=51{ }^{+}{ }^{+}$. defined as an increase in clinical pulmonary infection score more than two points. 

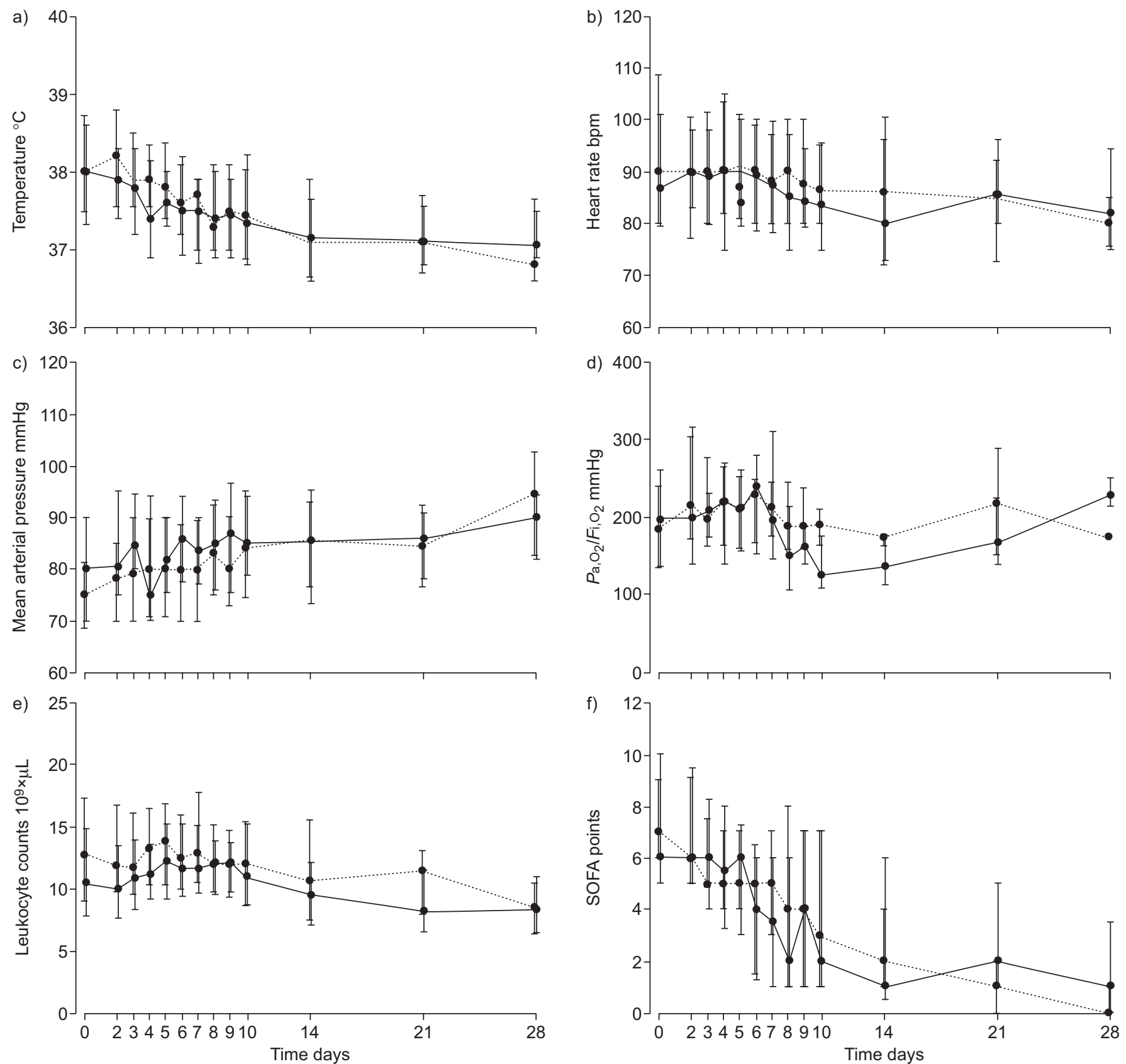

FIGURE 4. a-f) Physiological and functional score changes from day 0 to day 28 in the procalcitonin $(-)$ and control $(\cdots \cdots)$ group. $P a, O_{2}$ : arterial oxygen tension; $\mathrm{Fi}_{1} \mathrm{O}_{2}$ : inspiratory oxygen fraction; SOFA: sepsis-related organ failure assessment.

median mechanical ventilation- and ICU-free days alive were lower for patients with infection due to Gram-negative bacilli and MRSA. VAP-related clinical and radiological deterioration rate did not differ in both groups. Hospital discharge rate and post-discharge institution placement pattern was also comparable in both groups.

Deaths in the two groups were due to traumatic brain injury/ subarachnoid haemorrhage (four in the control and four in the procalcitonin group), septic shock (two in the control and one in the procalcitonin group), respiratory failure (three in the control and one in the procalcitonin group), cardiogenic shock (one in the control and one in the procalcitonin group), acute respiratory distress syndrome (one in the control group), multiorgan failure (one in the procalcitonin group) and acute liver failure (one in the control group). Patients who died during their hospitalisation had significantly higher levels of procalcitonin on admission than those who did not die (median (IQR) $1.29(0.21-2.00)$ versus $0.58(0.45-5.43), p=0.02)$, higher SAPS II scores (47.5 (43.4-55) versus 38 (31-47); $\mathrm{p}<0.001)$, and higher SOFA scores $(9(7-13.8)$ versus $6(6-9) ; \mathrm{p}=0.004)$. CPIS scores $(\mathrm{p}=0.935)$ and leukocyte counts $(\mathrm{p}=0.309)$ were similar in subjects who died and those who did not die.

Procalcitonin levels at VAP diagnosis correlated significantly with the increasing severity of the disease, as defined by the 
SAPS II score $\left(\mathrm{r}^{2}=0.358, \mathrm{p}<0.001\right)$, the number and the severity of acute organ failures related to ICU mortality $\left(\mathrm{r}^{2}=0.474\right.$, $\mathrm{p}<0.001$ ), and the presence or absence of organ dysfunctions and / or infection $\left(\mathrm{r}^{2}=0.254, \mathrm{p}=0.015\right)$. Circulating procalcitonin levels decreased similarly within 28 days of VAP onset in both randomised groups (fig. 4).

\section{DISCUSSION}

In this multicentre, randomised clinical trial we observed a benefit of incorporating procalcitonin into the antibiotic reduction strategy suggested by current ATS/IDSA guidelines. The average number of antibiotic-free days alive from day 1 to day 28 was $27 \%$ higher for patients who had been randomised to the procalcitionin group than for patients assigned to the control group. The procalcitonin-guided approach allowed to reduce the overall number of antibiotic-free days alive at 28 days by the same amount as reported by CHASTRE et al. [16] in his landmark trial, which randomised patients to receive 8 or 15 days of antibiotic therapy for VAP. Procalcitonin-guided strategy was able to significantly reduce the median duration of overall antibiotic treatment by one third, i.e. from 15 to 10 days. Despite precise guidelines based on strong evidence for reducing the duration of antibiotic treatment for VAP to 8 days [16], such practice has not been reported to be successfully translated in current clinical practice [21]. Accordingly, the long duration of antibiotic treatment in the control group in our study may be close to the current clinical practice in many centres. This seems to be true particularly for USA critical care centres, in which we observed a significantly longer median (IQR) overall duration of antibiotic therapy (15 (9-23) versus $11(6-17)$ days; $p=0.02)$ and antibiotic therapy for VAP (14.5 (7-20) versus 10 (5-15) days; $\mathrm{p}=0.027)$ as well as higher total number of antibiotic-agent days (26.6 (18.5-53.5) versus 12 (7-24) days; $\mathrm{p}<0.001$, respectively) compared with European centres. In contrast, the median overall antibiotic duration of 10 days in the procalcitonin-guided group, very close to the 8 days targeted by the recommendations for VAP, suggest that the use of this biomarker in addition to the other usual clinical, laboratory and radiological information may lead to effective bedside antibiotic discontinuation.

The benefit associated with procalcitonin-guidance was achieved by an increase in the number of antibiotic-free days alive after VAP onset. The primary end-point of this study, to evaluate whether a procalcitonin-guided strategy provides a real benefit in decreased overall antibiotic exposure, was accomplished by assessing the number of days without any antibiotic exposure. In this context, it represents a rather robust, i.e. conservative outcome.

The absence of differences in outcome parameters including the evolution of clinical and radiological signs and symptoms of pulmonary infection, the number of mechanical ventilationfree days alive, the number of ICU-free days alive, the length of hospital stay and overall mortality suggest that procalcitoninguided antibiotic reduction is not associated with a worse outcome in VAP. This also supports the concept that complications associated with long-term broad-spectrum antibiotic use may also have been avoided [7].

Currently, duration of antibiotic therapy in VAP is based on empirical rules and physician's preference [10, 16]. Deescalation of therapy is advocated for as an appropriate strategy for managing antibiotics for VAP. The de-escalation approach provides clinical balance between one extreme of using broad-spectrum, empiric antimicrobial agents as the sole treatment strategy and the other extreme of delaying the initiation of targeted therapy pending bacteriologic results [35]. The most recent ATS/IDSA guidelines emphasise the need for early and appropriate antibiotic therapy followed by deescalation whenever possible, based on culture and patient response [21]. Clinical strategies, based on evidence of clinical improvement, as defined by reduction in serial CPIS or improvement of the $\mathrm{Pa}_{\mathrm{a}} \mathrm{O}_{2} / \mathrm{Fi}, \mathrm{O} 2$ ratio at days 3 to 5 , have also succeeded in limiting antibiotic use in patients with mild VAP
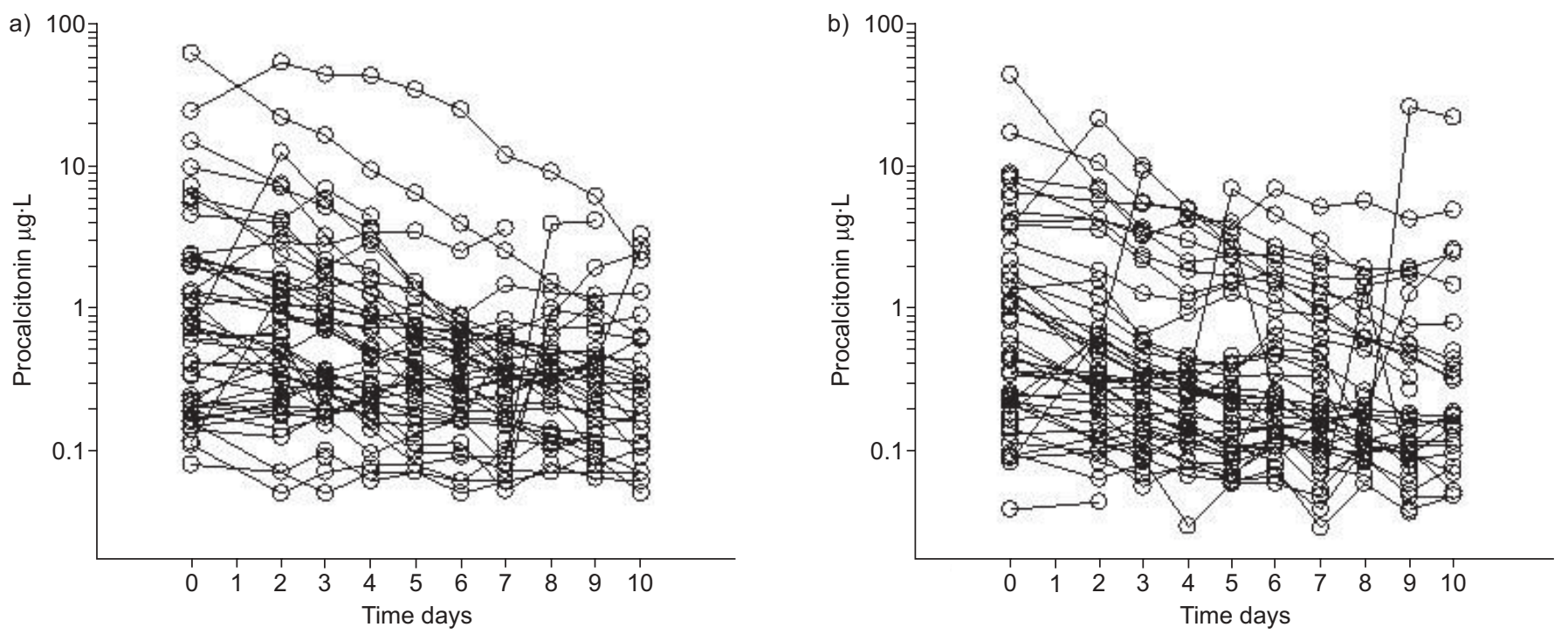

FIGURE 5. Serum procalcitonin changes from day 0 to day 28 in the a) control and b) procalcitonin groups. 
in the context of clinical trials $[15,17,18,36]$. Furthermore, cultures obtained from respiratory tract sampling consistently result in a change of antibiotic regimen allowing narrowing of antibiotics or monotherapy once microorganisms and theirs susceptibilities have been identified [37, 38]. Such practices were included in our strategies to reduce antibiotic exposure in both groups and may have contributed to our results.

It has been proposed that biomarkers might contribute to customise the duration of antibiotic treatment at a patient level, resulting in a greater tendency to limit or discontinue antibiotics $[19,30]$. Procalcitonin concentration closely parallels the severity and evolution of infection, including VAP, for which it has also a prognostic value [39-41]. This was also observed in our patients. We believe it is fair to assume that physicians taking care of patients with known (low) procalcitonin levels (procalcitonin group) tended to feel more confident in discontinuing antibiotic therapy than physicians taking care of patients, in whom procalcitonin levels were unknown (control group). Procalcitonin levels might have facilitated the discontinuation of one or all antimicrobial agents at the same time. This might explain why not only the number of antibiotic-free days alive was higher in patients randomised to the procalcitonin group, but also why patients randomised to the procalcitonin group received a smaller amount of antibiotic-agent days. In agreement with others, we observed a wide variation in serum procalcitonin concentrations at onset of VAP (fig. 5) [41, 42]. This suggests that individual VAP patients may require different lengths of antibiotic therapy according to different virulence of the causative microorganism and to individual host response to infection, factors potentially reflected by the evolution of serum procalcitonin levels [30].

The utility provided by the procalcitonin-guided strategy was more impressive in those patients with microbiologically diagnosed VAP. In our study, despite a high proportion of previous antibiotic exposure, VAP was microbiologically documented in $74 \%$ of the patients. This may again support that a procalcitonin-guided antibiotic discontinuation might provide an additional benefit to the currently applied microbiologically-guided de-escalation strategy.

In contrast to the impressive reduction in antibiotic exposure reported in patients admitted with community-acquired respiratory tract infections [27-29], we achieved only modest gains in antibiotic use for VAP. This may be due to additional difficulties in the interpretation of procalcitonin results in such ill patients, potentially related to higher proportion of previous systemic inflammatory response syndromes, multiorgan failure, previous infection and/or surgery, all known to raise the procalcitonin levels [25, 43].

Importantly, in the present study, procalcitonin kinetics assessed up to day 10 was used to support antibiotic discontinuation following $\geqslant 72 \mathrm{~h}$ empiric antimicrobial therapy for VAP. Hence, the results of this study do not allow primarily withholding of antibiotics based on procalcitonin levels at the time of VAP onset.

Our study has several limitations. First, we included a relatively small number of patients and our results should not be generalised to other settings such as hospital-acquired pneumonia and noncritically ill patients. It may not apply in the context of de-escalation strategies other then those suggested by ATS/IDSA guidelines. Secondly, these findings have to be validated in a larger cohort of patients, allowing sub-group analyses for selected populations, in whom infections may be more difficult to treat, e.g. severe chronic obstructive pulmonary disease and immunocompromised patients. Thirdly, our intervention was performed in a clinical setting, in which the final decision to de-escalate antibiotic treatment was left to the discretion of the attending physician. Thereby and in contrast to the study by CHASTRE et al. [16], physicians were not obliged to always conform to the study protocol. However, protocol overruling would result in a conservative bias, potentially underestimating the benefit of a procalcitonin-guided approach. Given the complexity of monitoring adherence to guidelines, we believe that it is fair to expect results of a study mimicking daily bedside practice to be more likely reproduced in a noninvestigation setting. Fourthly, we should emphasise that our trial was not designed to test the potential efficacy of a shorter duration of antibiotic treatment. In contrast, we demonstrated that a shorter duration of antibiotic therapy was not associated with a worse outcome. Finally, we did not conduct a formal cost-benefit study. Larger clinical trials are needed to explore the overall clinical and economic impact of the reduction of exposure to antibiotics in VAP patients. Strengths from our study are the multicentric design, the primary end-point evaluating the total number of antibiotic-free days alive up to 28 days irrespective of the indication for antibiotic therapy and the potential reproducibility of the results in daily clinical practice.

In conclusion, serum procalcitonin reduces antibiotic exposure in critically ill patients treated for VAP.

\section{CLINICAL TRIALS}

The study is registered in the Current Controlled Trials Database as "ProVAP"-study (ISRCTN61015974).

\section{SUPPORT STATEMENT}

D. Stolz was supported by grants from the Swiss National Foundation (Bern, Switzerland), the Margarete und Walter Liechtenstein Foundation (Basel, Switzerland) and the "Freiwillige Akademische Gesellschaft Basel" (Basel, Switzerland). N. Thakkar was supported by the Will Rogers Foundation (Toluca Lake, CA, USA). Additional funding was granted by the Clinic of Pulmonary Medicine, University Hospital Basel. Funding obtained from Brahms AG (Hennigsdorf, Germany) was used for assay material and logistics. The sponsors of this investigatorinitiated project had no involvement in design and conduct of the study, collection, management, analysis, and interpretation of the data, nor in the preparation, review, and approval of the manuscript or decision to submit the manuscript.

\section{STATEMENT OF INTEREST}

A statement of interest for the study itself can be obtained from www. erj.ersjournals.com/misc/statements.dtl

\section{ACKNOWLEDGEMENTS}

The current authors would like to thank A. Meyer (Clinic of Pulmonary Medicine and Respiraotry Cell Research, University Hospital, Basel, Switzerland), S. Heard, J. Longtine and K. Longtine (Dept of Anesthesiology, UMass Memorial Medical Center, University of Massachusetts, Worcester, MA, USA) for continuous support throughout the study, and A. Schötzau (Schötzau and Simmen Statistics, Basel) for statistical advice. 


\section{REFERENCES}

1 National Nosocomial Infections Surveillance (NNIS) System Report, data summary from January 1992 through June 2004, issued October 2004. Am J Infect Control 2004; 32: 470-485.

2 Vincent JL, Bihari DJ, Suter PM, et al. The prevalence of nosocomial infection in intensive care units in Europe. Results of the European Prevalence of Infection in Intensive Care (EPIC) Study. EPIC International Advisory Committee. JAMA 1995; 274: 639-644.

3 Kollef $\mathrm{MH}$. Ventilator-associated pneumonia. A multivariate analysis. JAMA 1993; 270: 1965-1970.

4 Chastre J, Fagon JY. Ventilator-associated pneumonia. Am J Respir Crit Care Med 2002; 165: 867-903.

5 Rello J, Ollendorf DA, Oster G, et al. Epidemiology and outcomes of ventilator-associated pneumonia in a large US database. Chest 2002; 122: 2115-2121.

6 Warren DK, Shukla SJ, Olsen MA, et al. Outcome and attributable cost of ventilator-associated pneumonia among intensive care unit patients in a suburban medical center. Crit Care Med 2003; 31: 1312-1317.

7 Soo Hoo GW, Wen YE, Nguyen TV, et al. Impact of clinical guidelines in the management of severe hospital-acquired pneumonia. Chest 2005; 128: 2778-2787.

8 Porzecanski I, Bowton DL. Diagnosis and treatment of ventilatorassociated pneumonia. Chest 2006; 130: 597-604.

9 Michaud S, Suzuki S, Harbarth S. Effect of design-related bias in studies of diagnostic tests for ventilator-associated pneumonia. Am J Respir Crit Care Med 2002; 166: 1320-1325.

10 Koenig SM, Truwit JD. Ventilator-associated pneumonia: diagnosis, treatment, and prevention. Clin Microbiol Rev 2006; 19: 637-657.

11 Rello J, Gallego M, Mariscal D, et al. The value of routine microbial investigation in ventilator-associated pneumonia. Am J Respir Crit Care Med 1997; 156: 196-200.

12 Fagon JY, Chastre J, Wolff M, et al. Invasive and noninvasive strategies for management of suspected ventilator-associated pneumonia. A randomized trial. Ann Intern Med 2000; 132: 621-630.

13 Kollef MH, Sherman G, Ward S, et al. Inadequate antimicrobial treatment of infections: a risk factor for hospital mortality among critically ill patients. Chest 1999; 115: 462-474.

14 Trouillet JL, Chastre J, Vuagnat A, et al. Ventilator-associated pneumonia caused by potentially drug-resistant bacteria. Am J Respir Crit Care Med 1998; 157: 531-539.

15 Ibrahim EH, Ward S, Sherman G, et al. Experience with a clinical guideline for the treatment of ventilator-associated pneumonia. Crit Care Med 2001; 29: 1109-1115.

16 Chastre J, Wolff M, Fagon JY, et al. Comparison of 8 vs 15 days of antibiotic therapy for ventilator-associated pneumonia in adults: a randomized trial. JAMA 2003; 290: 2588-2598.

17 Singh N, Rogers $\mathrm{P}$, Atwood CW, et al. Short-course empiric antibiotic therapy for patients with pulmonary infiltrates in the intensive care unit. A proposed solution for indiscriminate antibiotic prescription. Am J Respir Crit Care Med 2000; 162: 505-511.

18 Dennesen PJ, van der Ven AJ, Kessels AG, et al. Resolution of infectious parameters after antimicrobial therapy in patients with ventilator-associated pneumonia. Am J Respir Crit Care Med 2001; 163: 1371-1375.

19 Kollef $\mathrm{MH}$. Microbiological diagnosis of ventilator-associated pneumonia - using the data to optimize clincial outcomes. Am J Respir Crit Care Med 2008; 173: 1182-1183.

20 Kollef MH. Providing appropriate antimicrobial therapy in the intensive care unit: surveillance vs. de-escalation. Crit Care Med 2006; 34: 903-905.

21 Guidelines for the management of adults with hospital-acquired, ventilator-associated, and healthcare-associated pneumonia. Am J Respir Crit Care Med 2005; 171: 388-416.
22 Morrow LE, Shorr AF. The seven deadly sins of ventilatorassociated pneumonia. Chest 2008; 134: 225-226.

23 Muller B, Becker KL, Schachinger $\mathrm{H}$, et al. Calcitonin precursors are reliable markers of sepsis in a medical intensive care unit. Crit Care Med 2000; 28: 977-983.

24 Becker KL, Nylen ES, White JC, et al. Clinical review 167: Procalcitonin and the calcitonin gene family of peptides in inflammation, infection, and sepsis: a journey from calcitonin back to its precursors. J Clin Endocrinol Metab 2004; 89: 1512-1525.

25 Luyt CE, Combes A, Reynaud C, et al. Usefulness of procalcitonin for the diagnosis of ventilator-associated pneumonia. Intensive care Med 2008; 34: 1434-1440.

26 Pelosi P, Barassi A, Severgnini P, et al. Prognostic role of clinical and laboratory criteria to identify early ventilator-associated pneumonia in brain injury. Chest 2008; 134: 101-108.

27 Christ-Crain M, Jaccard-Stolz D, Bingisser R, et al. Effect of procalcitonin-guided treatment on antibiotic use and outcome in lower respiratory tract infections: cluster-randomised, singleblinded intervention trial. Lancet 2004; 363: 600-607.

28 Christ-Crain M, Stolz D, Bingisser R, et al. Procalcitonin guidance of antibiotic therapy in community-acquired pneumonia: a randomized trial. Am J Respir Crit Care Med 2006; 174: 84-93.

29 Stolz D, Christ-Crain M, Bingisser R, et al. Antibiotic treatment of exacerbations of COPD: a randomized, controlled trial comparing procalcitonin-guidance with standard therapy. Chest 2007; 131: 9-19.

30 Nobre V, Harbarth S, Graf JD, et al. Use of procalcitonin to shorten antibiotic treatment duration in septic patients: a randomized trial. Am J Respir Crit Care Med 2008; 177: 498-505.

31 Stolz D, Stulz A, Muller B, et al. BAL neutrophils, serum procalcitonin, and C-reactive protein to predict bacterial infection in the immunocompromised host. Chest 2007; 132: 504-514.

32 Altman DG, Schulz KF, Moher D, et al. The revised CONSORT statement for reporting randomized trials: explanation and elaboration. Ann Intern Med 2001; 134: 663-694.

33 BioMed CentralProcalcitonin for discontinuation of antibiotic therapy in clinically diagnosed ventilator associated pneumonia www.controlled-trials.com/ISRCTN61015974 Date last acessed: October 15, 2009.

34 Nylen ES, Muller B, Becker KL, et al. The future diagnostic role of procalcitonin levels: the need for improved sensitivity. Clin Infect Dis 2003; 36: 823-824.

35 Kollef MH, Morrow LE, Niederman MS, et al. Clinical characteristics and treatment patterns among patients with ventilatorassociated pneumonia. Chest 2006; 129: 1210-1218.

36 Luna CM, Blanzaco D, Niederman MS, et al. Resolution of ventilator-associated pneumonia: prospective evaluation of the clinical pulmonary infection score as an early clinical predictor of outcome. Crit Care Med 2003; 31: 676-682.

37 Shorr AF, Sherner JH, Jackson WL, et al. Invasive approaches to the diagnosis of ventilator-associated pneumonia: a meta-analysis. Crit Care Med 2005; 33: 46-53.

38 Rello J, Vidaur L, Sandiumenge A, et al. De-escalation therapy in ventilator-associated pneumonia. Crit Care Med 2004; 32: 21832190.

39 Wanner GA, Keel M, Steckholzer U, et al. Relationship between procalcitonin plasma levels and severity of injury, sepsis, organ failure, and mortality in injured patients. Crit Care Med 2000; 28 : 950-957.

40 Harbarth S, Holeckova K, Froidevaux C, et al. Diagnostic value of procalcitonin, interleukin-6, and interleukin-8 in critically ill patients admitted with suspected sepsis. Am J Respir Crit Care Med 2001; 164: 396-402.

41 Luyt CE, Guerin V, Combes A, et al. Procalcitonin kinetics as a prognostic marker of ventilator-associated pneumonia. Am J Respir Crit Care Med 2005; 171: 48-53. 
42 Seligman R, Meisner M, Lisboa TC, et al. Decreases in procalcitonin and $\mathrm{C}$-reactive protein are strong predictors of survival in ventilator-associated pneumonia. Crit Care 2006; 10: R125.
43 Suprin E, Camus C, Gacouin A, et al. Procalcitonin: a valuable indicator of infection in a medical ICU? Intensive Care Med 2000; 26: 1232-1238. 